\title{
Hypnotic General Anesthesia vs. Hypnotic Focused Analgesia in Preventing Pain and its Reflex Cardiovascular Effects
}

\author{
By Edoardo Casiglia* \\ Panagiota Rempelou ${ }^{*}$ \\ Valérie Tikhonoff \\ Federica Guidotti ${ }^{+}$ \\ Margherita Giacomello \\ Antonio M. Lapenta \\ Enrico Facco"
}

\begin{abstract}
Hypnotic focused analgesia (HFA) induces local anesthesia. The possibility to induce hypnotic general anesthesia (HGA) has never been investigated. An experimental study was conducted with 10 highly-hypnotizable young volunteers to compare the effects of HFA and those, if any, of HGA on pain perception and its reflex consequences. Pain tolerance was measured through the $m A$ of current necessary to induce maximum tolerable pain, and sympathetic discharge through the response of peripheral resistance $(P R)$. All experienced pain reduction at the maximum ESMN during HFA (-75\%, $p<0.0001)$ and HGA $(-87 \%, p<0.0001)$ in comparison to prehypnosis; 4 during HFA and 7 during HGA reported complete analgesia. The pain tolerance increased by $68 \%$ and by $101 \%(p=0.034)$, respectively. In prehypnosis, a $65.8 \%$ increase of the forearm $P R$ was observed during maximum tolerable pain. Lower variations of $P R$ were observed during HFA and no variations during HGA. HGA therefore exists and prevents pain perception and its consequences.
\end{abstract}

Keywords: Anesthesia, Human, Pain, Peripheral resistance, Sympathetic drive.

\section{Introduction}

The Laboratory of Experimental Hypnosis of the Department of Medicine of the University of Padua previously demonstrated that suggestions given in hypnosis induce behavioral and cognitive modifications that can be studied, repeated and measured in an experimental setting. Thermal (Casiglia et al. 2006) and situational (Casiglia et al. 1997a) hallucinations, analgesia for both non-trigeminal (Casiglia et al. 2007, Facco et al. 2013) and trigeminal (Facco et al. 2009, Facco et al. 2011) pain, hypnotic visuospatial neglect (Priftis et al.

\footnotetext{
* Department of Medicine, University of Padua, Italy.

Specialist in Internal Medicine, Department of Medicine, Hospital of Santorso, Italy.

$\$$ Department of Medicine, University Hospital of Padua, Italy.

${ }^{+}$Department of Medicine, University of Padua, Italy.

- Institute Franco Granone CIICS, Italy.

Institute Franco Granone CIICS, Italy.

- Studium Patavinum, University of Padua, Italy.
} 
2011), hypnotic alexia (Casiglia et al. 2010) and amusia for rhythm (Facco et al. 2014), hypnotic age regression (Giordano et al. 2012) and hypnotic increase in physical performance (Tikhonoff et al. 2012) were induced and quantified in a laboratory of haemodynamics with the peculiar tools of human physiology (Casiglia et al. 2012a). These studies showed that the effects of hypnosis, always real for the participant, are also real in an experimental context.

A special field of research is represented by the possibility to modulate pain through specific suggestions given in hypnosis (Kropotov et al. 1997, Chaves and Dworkin 1997, Holroyd 1996). Although the analgesic effect of these suggestions is well documented in highly hypnotizable subjects (highs), there is a lack of a theoretic basis about the mechanisms of hypnotic analgesia and the chain of events leading to analgesia remains quite uncertain. In particular, the fundamental question on whether hypnotic analgesia is due to mere subjective dissociation or to a real block of pain transmission is unanswered.

The procedures that are typical of cardio-physiology may be of help in this study (Kropotov et al. 1997). In fact, although point-rating visual analogical scales are commonly used to quantify pain (Mader et al. 2003), the nociceptive stimuli also have physiological effects that can be technologically measured (Casiglia et al. 2007). In the case of non-trigeminal (systemic) pain, these effects are represented by vasoconstriction with an increase of peripheral resistance and a reduction of cardiac output and peripheral flow.

Now, the measurement of such effects in a laboratory of physiology during hypnotic analgesia can answer the question about "dissociation or block". If hypnotic analgesia is accompanied by reduction of the reflex cardiovascular response to stress, the conclusion must be that pain is really blocked by hypnotic suggestions along the nervous ways from the area in pain to the brain; the reflex response to pain is in fact completely unconscious and not under the participant's consciousness. In other words, it cannot be voluntarily produced or simulated.

In previous studies we demonstrated that hypnotic focused analgesia (HFA) blocks not only perception of pain but also its hemodynamic consequences (Casiglia et al. 2007, Facco et al. 2009, Facco et al. 2011). It is about corresponding, in this respect, to chemically-induced local anesthesia (Facco et al. 2013).

The aim of the present study is to clarify whether it is possible to go beyond HFA, inducing through hypnotic suggestions a picture reproducing not only local analgesia but total-body analgesia, in the frame of what we decided to call hypnotic general anesthesia (HGA). 


\section{Materials and Methods}

\section{Study Subjects}

Ten healthy young volunteers aged $26.2 \pm 6.3$ years were studied. They were previously defined as eligible for hypnosis on the basis of a historical questionnaire and of a personal interview aimed for screening out subjects with a borderline personality, more prone to show unwanted effects during hypnotic dissociation. They were also defined as highs by means of the Harvard Group Scale of Hypnotic Susceptibility (De Pascalis et al. 2000, Younger 2005). Highs were chosen for the present pilot study as they tend to experience more evident pain inhibition following hypnotic procedures (Horton et al. 2004).

This research adheres to the principles of the Declaration of Helsinki. All subjects gave a written informed consent to the procedure. Each one was preliminary and personally informed about the aims, methods and possible risks of the procedure, and had the opportunity to ask all the questions that they considered necessary. The Local Ethics Committee approved the protocol.

\section{General Protocol of the Study}

\section{Hypnotic Procedures}

Some days before the experimental procedure, all subjects, in turn, individually underwent a classic hypnotic induction through verbal suggestions with cues of relax and well-being. Through the voice of an expert hypnotist, they were guided towards focusing their attention on a single idea, excluding other external or internal stimuli. A traditional induction was used, consisting in a brief enumeration coupled with suggestions of general well-being, eyelid heaviness, regular deep breathing, and staring at a point. After spontaneous eyelid closure was obtained, subjects were invited to concentrate on their own body from their feet to the head, whilst a feeling of heaviness and muscular relaxation was being suggested.

The verification of hypnosis was based on some signals, such as arm levitation, the easing of facial tension, the lower jaw dropping down with a mild opening of the mouth, and slower breathing rate. The analysis of these signals enabled the hypnotist to understand if the subjects were really hypnotized and it aimed at maintaining or modifying this condition by means of continuous appropriate suggestions.

The aim of this preparatory procedure was to establish a valid interpersonal relationship between the operator and the subject, in order to favor rapid and valid mono-ideism in the occasion of the following experimental sitting.

To reduce the time needed for induction, post-hypnotic conditioning was predisposed in all subjects leaving the conditioning: "When I..... [command], you will immediately fall in the same, profound trance condition you are now experiencing". 


\section{Experimental Session}

In the day chosen for the experiment, the subjects individually underwent, in pre-hypnotic basal conditions, an electrical stimulation of the right median nerve (ESMN) through a device giving square-wave direct current (DC) electric discharges at an increasing intensity and at a frequency of 2 stimuli/sec. As mentioned above, non trigeminal pain (like that involving ESMN) is expected to be accompanied by a reflex increase of peripheral resistance and a decrease of cardiac output (Casiglia et al. 2007). The subjects were kept in the supine posture for at least 30 minutes. In the meanwhile, the devices for hemodynamic monitoring were applied (Figure 1) to ensure the continuous measurement of the hemodynamic parameters specified below.

\section{Figure 1. Experimental Setting of the Study}

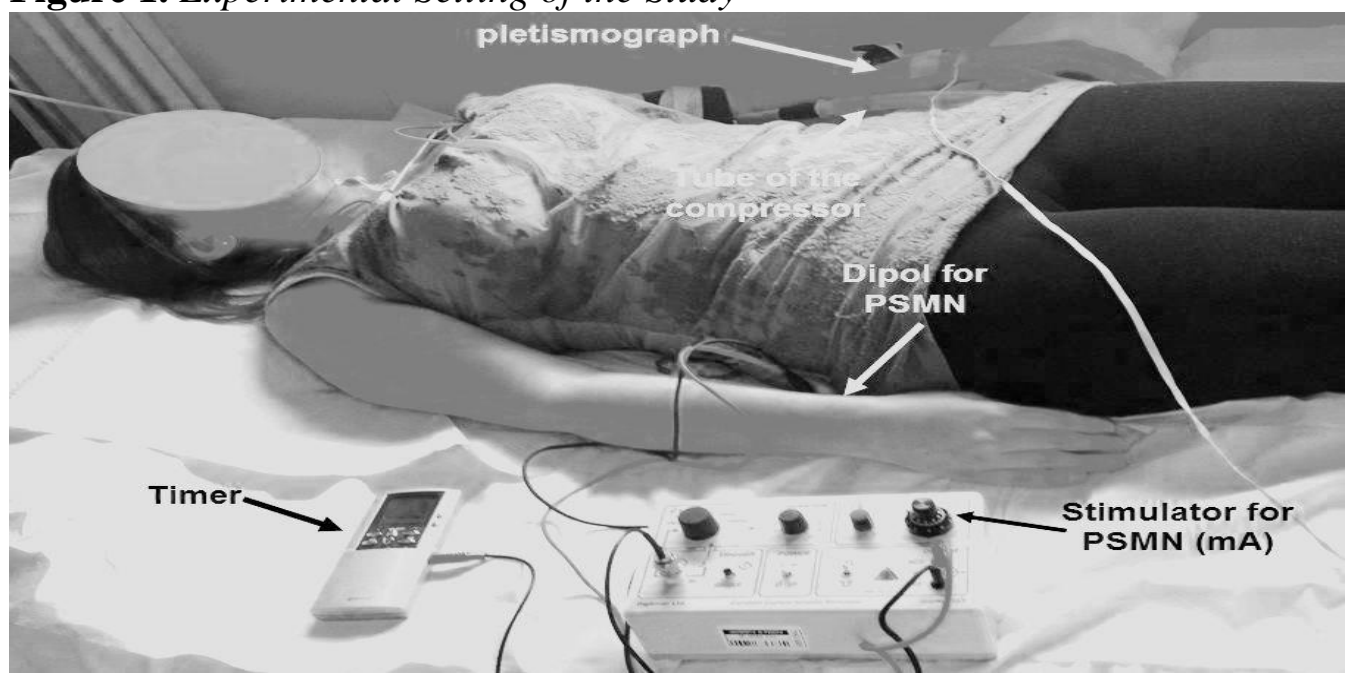

Source: Laboratory of Experimental Hypnosis, Department of Medicine, University of Padua, Italy.

After reaching hemodynamic stability, ESMN started from $0 \mathrm{~mA}$ and progressively increased to $0.2 \mathrm{~mA}$ per second until the participants reached a subjective feeling they could define as "pain" at hand thenar eminence. This intensity was recorded and, after 1-minute of constant ESMN, the hemodynamic parameters described below were measured. In a second phase following the previous one, the DC intensity was increased by $20 \%$ and the hemodynamic parameters were recorded once more after 1-minute of ESMN. In a third phase, the DC intensity was further increased by $0.2 \mathrm{~mA}$ per second until the participant said the pain became "intolerable". This intensity was recorded as well and, after 1-minute ESMN, the hemodynamic parameters described below were measured once more. The EMSN was then stopped and the pain immediately ceased. The maximum pain reached during ESMN was therefore quantified in two ways: 1) by a 0-to-10 subjective rating scale immediately after the end of the procedure (subjective pain tolerance threshold), and 2) by measuring the maximum tolerable DC intensity (objective pain tolerance threshold). 
In the same day, the same subjects were asked to repeat the same procedure while undergoing HFA and HGA in a random sequence.

For HFA, once valid neutral hypnosis was reached, specific suggestions aimed at obtaining right hand analgesia were administered. In detail, it was suggested that the right hand was insensitive to pain and that no pain sensation could originate from it. Aim of this procedure was to produce analgesia by focusing attention on/from the hand receiving the painful stimulation. The pain intensity (0-to-10 rating visual scale) and pain tolerance threshold (maximum tolerable DC intensity in $\mathrm{mA}$ ) were recorded as previously described and hemodynamic parameters were continuously monitored before and during the entire painful procedure.

The DC intensity administered at threshold and at threshold $+20 \%$ phases was the same as in pre-hypnotic conditions, while that administered to

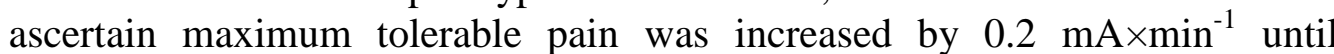
subjects said to have reached the maximum level of tolerable pain and wanted to cease the ESMN. This intensity was recorded for determination of the objective pain tolerance threshold.

For patients who developed complete analgesia and resulted to be insensitive to pain during this phase, it was decided a priori to stop the test at the $10^{\text {th }} \mathrm{min}$. During hypnosis, continuous suggestions of well being were given. The subject's usual consciousness was then gradually restored and the participant was de-hypnotized.

For HGA, any effort was made in order to reproduce the conditions that are typical of general anesthesia (Figure 2).

Figure 2. Experimental Model Aimed at Reproducing Via Hypnotic General Anesthesia the Items that Are Typical of Spontaneous-Breathing Pharmacological General Anesthesia

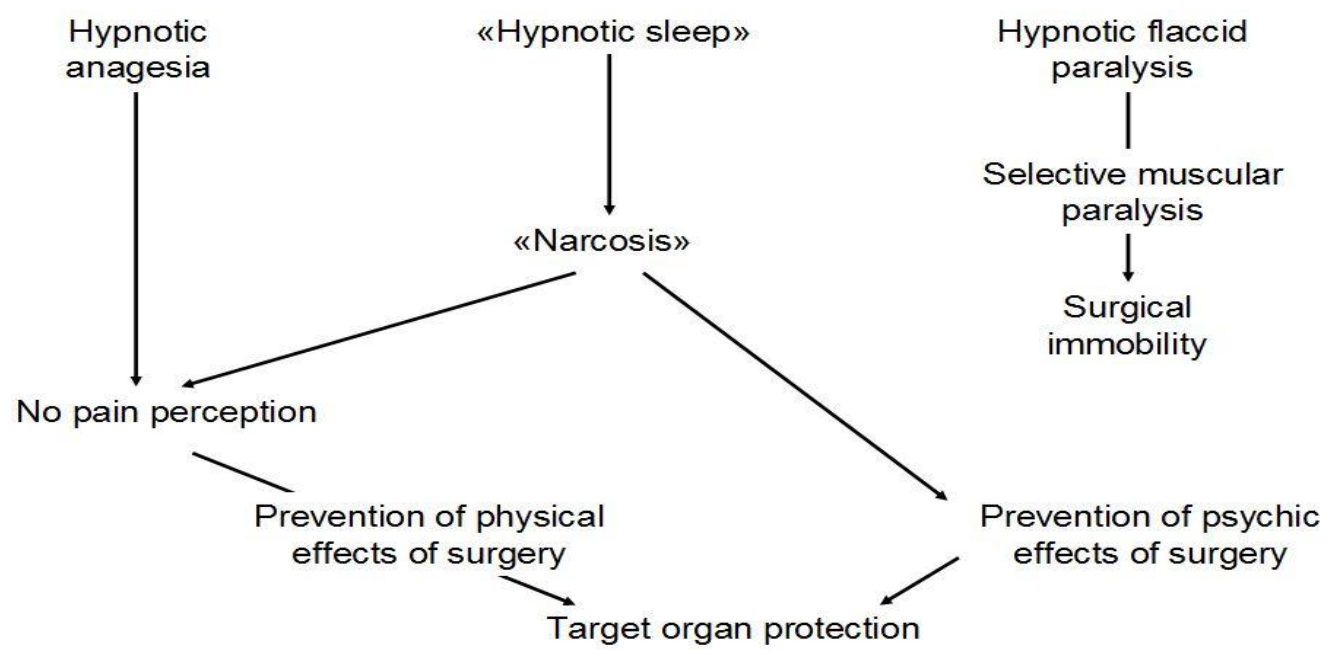

Source: This model was used for the study presented herein by the Laboratory of Experimental Hypnosis, Department of Medicine, University of Padua, Italy.

Suggestion of analgesia was extended to the whole body, reproducing pharmacological analgesia. Suggestions of flaccid paralysis were given for all 
muscles but the respiratory ones, like in spontaneous-breathing chemicallyinduced general anesthesia. Hypnotic relaxation was suggested to reproduce the sedation obtained in general anesthesia through benzodiazepines. Profound "hypnotic sleep" was suggested to reproduce the narcosis that is obtained in general anesthesia by means of barbiturates. During HGA, the participant did not know where the painful stimuli would be given. DC intensity administered at threshold and at threshold $+20 \%$ phases was the same as in pre-hypnotic conditions. While that administered to ascertain maximum tolerable pain was increased by $0.2 \mathrm{mAxmin}^{-1}$ until the subjects said to have reached the maximum level of tolerable pain and wanted to cease the ESMN. This intensity was recorded for determination of the objective pain tolerance threshold.

During hypnosis, continuous suggestions of well being were given. The subject's usual consciousness was then gradually restored and the participant was de-hypnotized.

The following cardiovascular parameters were monitored in pre-hypnotic basal conditions and during HFA and HGA.

\section{Hemodynamic Measurements}

Blood pressure (in $\mathrm{mmHg}$ ) was measured by an Omron 705 IT device (Omron Europe, Hoofddorp, The Netherlands). Pulse pressure (in $\mathrm{mmHg}$ ) was calculated as systolic minus diastolic, and mean blood pressure (in $\mathrm{mmHg}$ ) as diastolic $+1 / 3$ pulse pressure. In 6 subjects, the blood flow indexed for the volume of tissue (in $\mathrm{ml} \times \mathrm{min}^{-1} \times \mathrm{dl}^{-1}$ ) was measured from the left forearm with a strain-gauge plethysmograph (Angioflow, Microlab Electronics, Padova, Italy). This method, which is validated and largely used by our research group (Casiglia et al. 2006, Casiglia et al. 1997a, Casiglia et al. 2007, Giordano et al. 2012, Kropotov et al. 1997, Casiglia et al. 2012b, Casiglia et al. 1995, Casiglia et al. 1998a, Casiglia et al. 1997b, Casiglia et al. 1998b, Casiglia et al. 1999, Casiglia et al. 1998c, Casiglia et al. 1991), entails periodic occlusion of venous outflow by a cuff automatically inflated at over-venous and under-diastolic pressure and the measurement of arm volume by indium-gallium-in-silicone strain-gauge. In such conditions, segmental blood inflow is proportional to arterial flow and is automatically calculated by the inner software. Left forearm arterial peripheral resistance was calculated (in $\mathrm{mmHg} \times \mathrm{min} \times \mathrm{dl} \times \mathrm{ml}^{-1}$ ) from the mean blood pressure/arterial flow ratio. The aim of these measurements was to ascertain whether the ESMN was accompanied by a sympathetic drive discharge leading to vasoconstriction, or on the contrary this sympathetic response to pain was reduced or absent during HFA and/or HGA. The DC intensity administered was recorded in order to quantify (in $\mathrm{mA}$ ) objective pain tolerance.

Continuous variables were expressed as mean \pm standard deviation and compared with an intra-subject paired $t$ test in square-Latin design. 95\% confidence intervals (CI) were shown when proper. The null hypothesis was rejected for a probability $(p)$ of 0.05 or less. 


\section{Results}

After de-hypnotization, all subjects stated to feel perfectly well, to be calmer than before the experiment, and to have experienced a reduction in pain perception at the maximum tolerable ESMN during both HFA and HGA in comparison to pre-hypnotic conditions (Figure 3).

Maximum pain perception was in average $9.5 \pm 0.6$ in pre-hypnotic conditions, $2.4 \pm 3.0$ during HFA $(-75 \%$ vs. pre-hypnosis, $\mathrm{p}<0.0001)$ and $1.2 \pm 2.5$ during HGA ( $-87 \%$ vs. pre-hypnosis, $\mathrm{p}<0.0002$ vs. pre-hypnosis, non significant difference vs. HFA). Four subjects over 10 during HFA and 7 over 10 during HGA reported complete analgesia (visual scale score equal to 0 ).

Figure 3. Maximum Pain Perception in Pre-Hypnotic Basal Conditions and Iin Conditions of Hypnotic Focused Analgesia (HFA) and Hypnotic General Anesthesia (HGA) in 10 Highly-Hypnotizable Normal Subjects

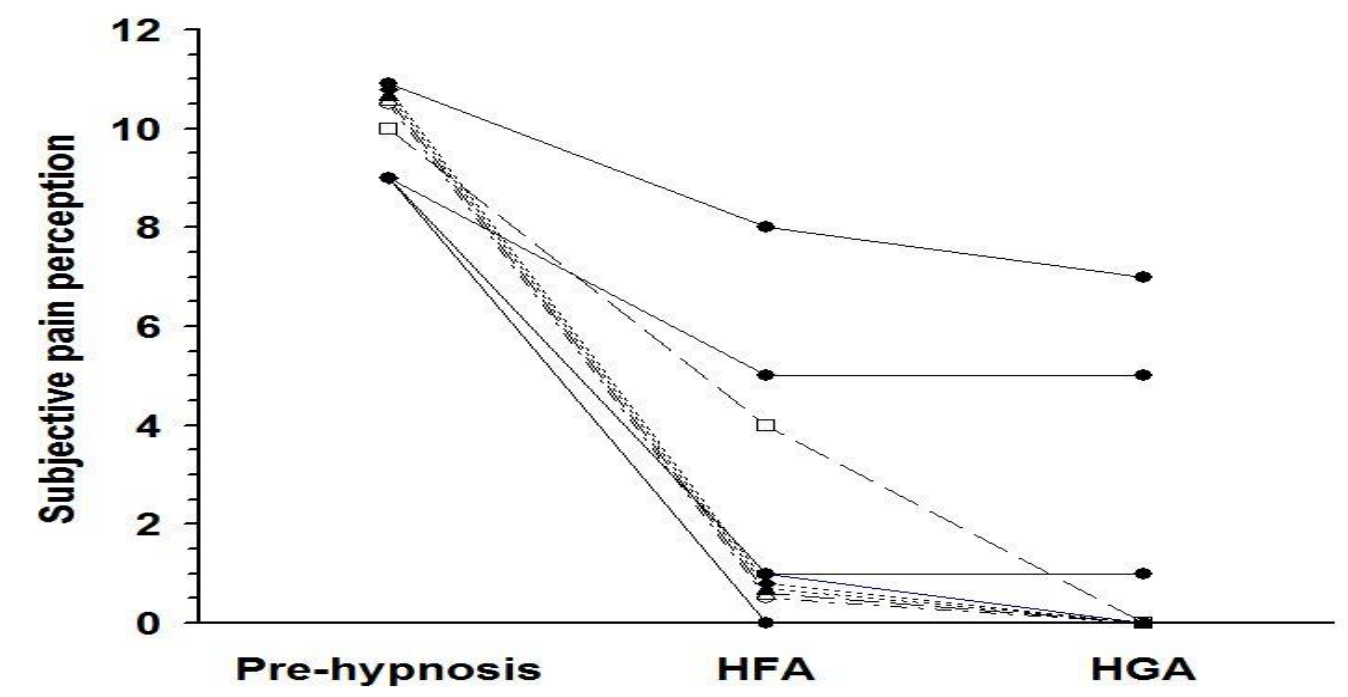

Source: Results from the Laboratory of Experimental Hypnosis, Department of Medicine, University of Padua, Italy.

The increase of tolerance to ESMN (maximum pain vs. pain threshold) was 26.9 \pm 27.0 (CI 5.5-48.4) $\mathrm{mA}$ in pre-hypnosis, 45.2 \pm 46.8 (CI 11.7-78.6) $\mathrm{mA}$ in HFA (+68\% vs. pre-hypnosis), and 54.1 \pm 53.4 (CI 15.8-92.3) $\mathrm{mA}$ in HGA $(+101 \%$ vs. pre-hypnosis, $\mathrm{p}=0.034)$.

The trend of hemodynamic parameters during ESMN in the three experimental conditions is shown in Table 1. In pre-hypnotic basal conditions, increasing values of ESMN were associated to significant increase of the sympathetic drive, with a significant peak of peripheral resistance $(+65.8 \%$, $\mathrm{p}<0.05)$ and a mirroring decrease of peripheral flow $(-17.3 \%, \mathrm{p}<0.05)$ in correspondence to the maximum tolerable pain (Figure 4). Lower and non significant variations of these two parameters were observed during HFA and there were practically no variations during HGA (Figure 4). 
Vol. 3, No. 2 Casiglia et al.: Hypnotic General Anesthesia vs. Hypnotic Focused ...

Table 1. Hemodynamic Parameters Recorded in Pre-Hypnotic Basal Conditions and During Hypnotic Focused Analgesia and of Hypnotic General Anesthesia

\begin{tabular}{|c|c|c|c|c|}
\hline & \multicolumn{4}{|c|}{ Pre-hypnotic basal conditions } \\
\hline & Baseline & Pain threshold & $\begin{array}{l}\text { Threshold } \\
+20 \%\end{array}$ & $\begin{array}{l}\text { Maximum } \\
\text { pain }\end{array}$ \\
\hline Leg PR (UR) & $26.3 \pm 8.3$ & $32.8 \pm 7.7$ & $33.7 \pm 10.0$ & $43.6 \pm 19.5^{*}$ \\
\hline Leg RF (UF) & $3.4 \pm 1.1$ & $2.6 \pm 0.9$ & $2.6 \pm 0.8$ & $2.2 \pm 0.7 *$ \\
\hline SBP (mmHg) & $112.7 \pm 15.2$ & $117.5 \pm 17.7$ & $117.4 \pm 19.0$ & $117.4 \pm 17.0$ \\
\hline DBP (mmHg) & $70.1 \pm 10.5$ & $70.9 \pm 11.4$ & $72.2 \pm 12.2$ & $72.2 \pm 12.2$ \\
\hline MBP (mmHg) & $84.3 \pm 11.5$ & $86.4 \pm 13.1$ & $87.3 \pm 13.6$ & $87.3 \pm 13.6$ \\
\hline HR $\left(\mathbf{b} \times \mathrm{min}^{-1}\right)$ & $80.2 \pm 17.9$ & $83.5 \pm 21.7$ & $82.5 \pm 19.4$ & $82.5 \pm 19.4$ \\
\hline \multirow[t]{3}{*}{ CO $\left(1 \times \min ^{-1}\right)$} & $5.2 \pm 2.9$ & $4.3 \pm 3.0$ & $4.2 \pm 2.6$ & $4.3 \pm 3.2$ \\
\hline & \multicolumn{4}{|c|}{ Hypnotic focused analgesia (HFA) } \\
\hline & Baseline & Pain threshold & $\begin{array}{l}\text { Threshold } \\
+20 \%\end{array}$ & $\begin{array}{l}\text { Maximum } \\
\text { pain }\end{array}$ \\
\hline Leg PR (UR) & $32.0 \pm 14.6$ & $32.6 \pm 13.8$ & $34.0 \pm 17.5$ & $37.2 \pm 16.7$ \\
\hline Leg RF (UF) & $3.2 \pm 1.5$ & $2.9 \pm 1.2$ & $3.1 \pm 1.7$ & $2.8 \pm 1.2$ \\
\hline SBP (mmHg) & $117.9 \pm 15.3$ & $116.6 \pm 15.0$ & $117.7 \pm 15.7$ & $120.8 \pm 15.7$ \\
\hline DBP (mmHg) & $73.4 \pm 11.1$ & $74.3 \pm 11.6$ & $73.2 \pm 10.6$ & $76.2 \pm 11.6$ \\
\hline MBP (mmHg) & $88.2 \pm 11.7$ & $88.4 \pm 12.2$ & $88.0 \pm 11.6$ & $91.1 \pm 12.0$ \\
\hline HR $\left(\mathbf{b} \times \min ^{-1}\right)$ & $83.6 \pm 20.2$ & $84.0 \pm 20.2$ & $84.8 \pm 23.4$ & $89.6 \pm 21.6$ \\
\hline \multirow[t]{3}{*}{ CO $\left(\right.$ l $\left.\times \mathrm{min}^{-1}\right)$} & $4.0 \pm 2.2$ & $4.9 \pm 2.1$ & $3.1 \pm 1.7$ & $2.8 \pm 1.2$ \\
\hline & \multicolumn{4}{|c|}{ Hypnotic general anesthesia (HGA) } \\
\hline & Baseline & Pain threshold & $\begin{array}{l}\text { Threshold } \\
+20 \%\end{array}$ & $\begin{array}{l}\text { Maximum } \\
\text { pain }\end{array}$ \\
\hline Leg PR (UR) & $33.2 \pm 15.5$ & $38.9 \pm 18.4$ & $34.9 \pm 16.2$ & $35.1 \pm 15.1$ \\
\hline Leg RF (UF) & $2.9 \pm 1.3$ & $2.5 \pm 1.1$ & $1.9 \pm 1.5$ & $2.8 \pm 1.0$ \\
\hline SBP (mmHg) & $114.6 \pm 14.0$ & $115.8 \pm 14.9$ & $115.8 \pm 16.9$ & $119.2 \pm 11.4$ \\
\hline DBP (mmHg) & $71.9 \pm 10.7$ & $72.1 \pm 11.3$ & $73.9 \pm 11.6$ & $74.3 \pm 11.4$ \\
\hline MBP (mmHg) & $86.1 \pm 11.3$ & $86.7 \pm 11.9$ & $87.9 \pm 12.8$ & $89.3 \pm 11.9$ \\
\hline HR $\left(b \times \min ^{-1}\right)$ & $81.1 \pm 19.8$ & $81.1 \pm 21.6$ & $79.0 \pm 21.3$ & $85.7 \pm 17.6$ \\
\hline $\mathrm{CO}\left(\mathrm{l} \times \mathrm{min}^{-1}\right)$ & $4.1 \pm 2.5$ & $3.6 \pm 2.0$ & $4.4 \pm 3.3$ & $5.4 \pm 3.6$ \\
\hline
\end{tabular}

Note: ${ }^{*} \mathrm{p}<0.05$ vs. baseline in pre-hypnotic conditions. PR: peripheral arteriolar resistance; RF: arterial rest flow; SBP, DBP and MBP: systolic, diastolic and mean arterial blood pressure; HR: heart rate; CO: cardiac output; UR: units of arteriolar resistance $\left(\mathrm{mmHg} \times \min \times \mathrm{dl} \times \mathrm{ml}^{-1}\right)$; UF: units of arterial flow $\left(\mathrm{ml} \times \mathrm{min}^{-1} \times \mathrm{dl}^{-1}\right)$.

Source: Results from the Laboratory of Experimental Hypnosis, Department of Medicine, University of Padua, Italy. 
Figure 4. Percent Variations of Leg Arteriolar Resistance and of Leg Rest Arterial Flow at Maximum Pain Perception in Pre-Hypnotic Basal Conditions and in Conditions of Hypnotic Focused Analgesia (HFA) and Hypnotic General Anesthesia (HGA) in 10 Highly-Hypnotizable Normal Subjects

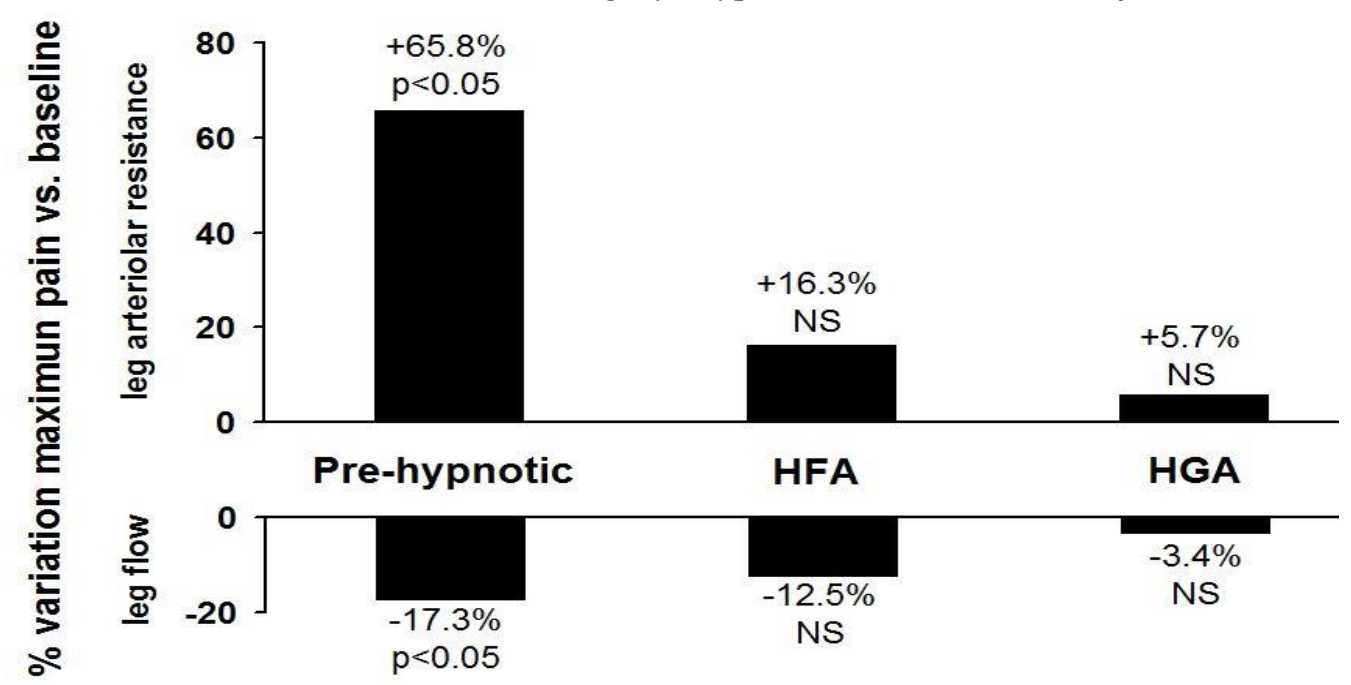

Note: NS: No significant difference.

Source: Results from the Laboratory of Experimental Hypnosis, Department of Medicine, University of Padua, Italy.

\section{Discussion}

Our data confirm the results we previously obtained using the cold pressor test as a pain maneuver (Casiglia et al. 2007). In fact, the perception of pain measured at the peak of ESMN with the visual rating scale (the so called subjective pain tolerance) was $87 \%$ lower than in pre-hypnotic conditions and abolished in $40 \%$ of the cases. Furthermore, the excess DC discharge intensity necessary to induce the maximum tolerable pain in comparison to the pain threshold (the so called objective pain tolerance) was $68 \%$ higher in HFA than in pre-hypnotic conditions.

Not only this but the vasoconstriction that, in pre-hypnotic basal conditions, accompanied the hemodynamic response to ESMN was strongly reduced during HFA, reflecting a parallel reduction of the pain-induced stress. HFA was therefore able to limit not only the subjective perception of pain and its objective tolerance, but also its reflex cardiovascular consequences. This is not novel, as our group showed in the past that the HFA is not sustained by a mere dissociation, but rather by a real block of painful stimuli according to the theory of gate control.

Up to date, hypnotic analgesia had been studied only in terms of HFA, functionally equivalent to chemical (pharmacological) local anesthesia. In the present study we also wanted to analyze the effects of the hypnotic suggestions reproducing general anesthesia. As known, general anesthesia is represented by analgesia (usually obtained with opioid agents), by narcosis (obtained for instance with a gas or an intravenous drug), by surgical immobility (obtained 
with curarization and requiring artificial ventilation), and by retrograde amnesia (obtained with benzodiazepines). If curarization is omitted, spontaneous-breathing general anesthesia (also called deep sedation) is produced. Apart from pain relief, the final aim of all these procedures is the prevention of organ damage.

According to the results shown herein, HGA (obtained without any drugs) qualified itself as a very effective analgesic tool, as pain perception (subjective pain tolerance) at maximum ESMN was $89 \%$ lower than in pre-hypnotic conditions and completely abolished in $70 \%$ of cases. Furthermore, the excess DC discharge intensity necessary to induce the maximum tolerable pain in comparison to pain threshold (objective pain tolerance) was $101 \%$ higher in HGA than in pre-hypnotic conditions. The reflex vasoconstriction accompanying pain was also nullified, with preservation of peripheral flow. HGA was therefore able not only to reduce (and in some cases to abolish) subjective pain perception, but also to prevent its reflex cardiovascular effects, preserving target organs from stress ischemia during the pain procedure. This pattern is commonly observed in pharmacologically-induced general anesthesia, where it is the resultant of the combination of analgesia, narcosis and neuromuscular paralysis. The ability of hypnosis to mime the situations of physical reality is confirmed once again by the present experiment.

As known, the mechanisms of hypnotic analgesia are uncertain. Analgesia could be the result of mere dissociation, or, on the contrary, it could imply a real block of painful stimuli along the nervous ways. The results of the present study discriminate between these two hypotheses. In fact, if pain were simply dissociated from consciousness, its reflex cardiovascular effects should be fully maintained, as the sympathetic mechanisms responsible for reflex vasoconstriction act at a lower level than subjective consciousness (where dissociation happens). Lack of increase of arteriolar resistance in the case of HFA and especially of HGA therefore reflects a real block of painful stimuli at a certain level of the sensitive system. In other words, the results of the present study, like those we previously obtained in our laboratory with a different type of painful stimulation (Casiglia et al. 2007), are in agreement with the models considering the reduction of nociceptive stimuli during hypnotic analgesia (Kiernan et al. 1995, Sandrini et al. 2000).

This reduction can happen only by an object of speculation, gate control being a possible explanation. The gate control theory postulates a gating system at the dorsal horn of the spinal cord modulating the transmission of noxious signaling (Melzack and Wall 1965), as well as selective cognitive processes transmitted through descending fibers modulating properties of the spinal cord gating. Hypnosis could affect the system processing nociception and in fact changes in the nociceptive spinal cord reflex R-III that is experimentally observed by other authors during hypnotic analgesia (Kiernan et al. 1995).

It is also conceivable that hypnosis operates on multiple levels: reduction of R-III at the spinal cord, reduction in pain feeling over and beyond R-III related to brain mechanisms preventing awareness of pain, and reduction in 
unpleasantness related to selective reduction in the affective dimension as a consequence of reinterpretation of meanings associated with the painful sensation (Kiernan et al. 1995).

In this view, hypnotic analgesia could modulate the progression of pain at different levels and in different areas of the nervous system. Pain is in fact a multi-dimensional experience involving sensory, dissociative, motivational, emotional and cognitive factors, and hypnosis could act by down-regulating the input of the painful stimuli by activating $\delta$ and $\mathrm{C}$ fibers, so reducing the sympathetic arousal too (Casiglia et al. 2007): at a spinal level hypnosis could reduce the sympathetic reflexes, at a cortical level it could modulate perception and the affective dimension of pain, and finally controlled cognitive processes could be associated to a supervising attentive system involving frontotemporal cortex and the limbic system.

On the contrary, we must reject the so-called dissociative models (following which pain could be registered but would remain dissociated from conscious and emotional awareness because of an amnesia-like barrier) and the social-cognitive models (following which hypnotic analgesia would be similar to waking analgesia, consisting of distracting attention from the pain by using goal-directed fantasies) as the sole mechanisms of HFA and HGA. In fact in the above mentioned conditions the reflex cardiovascular response to pain should be fully maintained, which is not the case in our experience.

Thanks to its attitudes, hypnotic analgesia (even when extended to the whole body in the context of HGA, miming spontaneous-breathing pharmacological general anesthesia) can be of help in preventing surgical pain and in protecting target organs (such as heart and brain) from unwanted reflex cardiovascular consequences of nociception.

Both HFA and HGA have a low cost and do not need a recovery period, appearing more advantageous than chemical anesthesia in these respects. In highs, the effects of HGA are those of spontaneous-breathing general anesthesia or profound sedation with analgesia. It could be of particular help in dentistry, in small surgery, in plastic surgery (particularly for burns management), and in painful or fastidious procedures such as endoscopy or biopsy. Since they do not need intubation and ventilation, both HFA and HGA do not impose the presence of an anesthetist and can be administered with safety by a doctor or a nurse expert in hypnotism.

The main strength of the present study is that it demonstrated for the first time that HGA is possible and effective. Its main limitation is that it was limited to highs, while scarcely hypnotizable subjects were deliberately excluded from the protocol. Another limitation is that the analysis is limited to 10 subjects. On the other hand, this is nothing more than a pilot study aimed at demonstrating the efficacy of HGA and at comparing HGA and HFA in an experimental setting. The results must be confirmed in larger samples. If the mechanisms of HFA and HGA is really attributable to a block of painful stimuli in the frame of the gate control systems as suggested in the present paper, they need to be confirmed by neurophysiological tests. 
Vol. 3, No. 2 Casiglia et al.: Hypnotic General Anesthesia vs. Hypnotic Focused ...

\section{Conclusions}

As previously reported in medical literature, demonstrated in the past by our research group (Casiglia et al. 2007, Facco et al. 2013, Facco et al. 2009, Facco et al. 2011) and shown in the present paper, suggestions of analgesia given in hypnosis are very effective in reducing not only the subjective perception of pain (Casiglia et al. 2007, Facco et al. 2013, Facco et al. 2009, Facco et al. 2011) but also its reflex cardiovascular effects (Casiglia et al. 2007, Facco et al. 2009, Facco et al. 2011) in highs.

This is evident in the setting represented by the more commonly used HFA (Casiglia et al. 2007, Facco et al. 2013), and even more evident in that, described herein for the first time, represented by HGA.

HGA, obtained by adding hypnotic analgesia, "hypnotic sleep", hypnotic muscular paralysis and hypnotic amnesia perfectly reproduce the picture of analgesia + narcosis + paralysis + retrograde amnesia that is typical of chemical (pharmacological) general anesthesia, with the difference that intubation and artificial ventilation are not necessary. In fact, spontaneous breathing is -of course- always maintained during hypnosis.

This makes HGA similar to spontaneous-breathing general anesthesia, also called profound sedation. This hypnotic syndrome may be very useful in a variety of situations in which pharmacological general anesthesia is difficult to apply, too expensive or contraindicated, such as small surgery, dentistry, treatment of burning, invasive or nagging diagnostic maneuvers (e.g. endoscopy, hysteroscopy, treatment of bone fractures), application of cardiac pacemakers, application and removal of nasal packing, biopsy, magnetic resonance in phobics and so on. In these conditions, HGA has the additional advantage not to require the presence of an anesthetist and not to lead to unwanted respiratory depression.

\section{References}

Casiglia E, Tikhonoff V, Giordano N, Andreatta E, Re-Galdo G, Tosello MT, Rossi AM, Bordin D, Giacomello M, Facco E (2012a) Measured outcomes with hypnosis as an experimental tool in a cardiovascular physiology laboratory. International Journal of Clinical and Experimental Hypnosis 60: 241-261.

Casiglia E, Tikhonoff V, Giordano N, Regaldo G, Facco E, Marchetti P, Schiff S, Tosello MT, Giacomello M, Rossi AM, De Lazzari F, Palatini P, Amodio P (2012b) Relaxation versus fractionation as hypnotic deepening: do they differ in physiological changes? International Jounal of Clinical and Experimental Hypnosis 60: 338-355.

Casiglia E, Schiff S, Facco E, Gabbana A, Tikhonoff V, Schiavon L, Bascelli A, Avdia $\underline{\text { M}}$, Tosello MT, Rossi AM, Haxhi Nasto H, Guidotti F, Giacomello M, Amodio

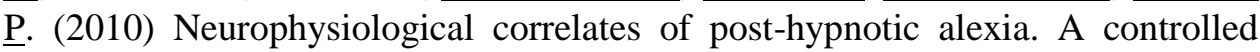
study with Stroop test. American Journal of Clinical Hypnosis 52: 219-233.

Casiglia E, Schiavon L, Tikhonoff V, Haxhi Nasto H, Azzi M, Empelo P, Giacomello M, Bolzon M, Bascelli A, Scarpa R, Lapenta AM, Rossi AM (2007) Hypnosis 
prevents the cardiovascular response to cold pressor test. American Journal of Clinical Hypnosis 49: 255-266.

Casiglia E, Rossi A, Tikhonoff V, Scarpa R, Tibaldeschi G., Giacomello M, Canna P, Schiavon L, Rizzato A, Lapenta AM (2006) Local and systemic vasodilation following hypnotic suggestion of warm tub bathing. International Journal of Psychophysiology 62: 60-65.

Casiglia E, Pizziol A, Piacentini F, Biasin R, Onesto C, Tikhonoff V, Prati V, Palatini $\mathrm{P}$, Pessina AC (1999) 24-hour leg and forearm haemodynamics in transected spinal cord subjects. Cardiovascular Researsch 41: 312-316.

Casiglia E, Pavan L, Marcato L, Leopardi M, Pizziol A, Salvador P, Zuin R, Pessina AC (1998a) Subjects with obstructive pulmonary disease tend to be chronically vasodilated. Clinical Science 95: 287-294.

Casiglia E, Palatini P, Ginocchio G, Biasin R, Pavan L, Pessina AC (1998b) Leg versus forearm flow: $24 \mathrm{~h}$ monitoring in 14 normotensive subjects and in 14 agematched hypertensive patients confined to bed. American Journal of Hypertension 11: 190-195.

Casiglia E, Mazza A, Ginocchio G, Onesto C, Pessina AC, Rossi A, Cavatton G, Marotti A (1997a) Haemodynamics following real and hypnosis-simulated phlebotomy. American Journal of Clinical Hypnosis 4: 368-375.

Casiglia E, Pessina AC, Bongiovì S, Michieletto M, Ginocchio G, Biasin R, Pizziol A, Palatini P (1997b) Central and peripheral hemodynamics during and after longlasting two-leg exercise in borderline hypertensive males. International Journal of Sports Cardiology 6: 133-138.

Casiglia E, Petucco S, Pessina AC (1995) Antihypertensive efficacy of amlodipine and enalapril and effects on peripheral blood flow in patients with essential hypertension and intermittent claudication. Clinical Drug Investigation 11(Suppl.): 97-101.

Casiglia E, Staessen J, Ginocchio G, Onesto C, Pegoraro L, Pizziol A, Palatini P, Pessina AC (1998c) Characterisation of hypertensive patients according to $24 \mathrm{~h}$ peripheral resistance. Japanese Heart Journal 39: 355-362.

Casiglia E, Bongiovì S, Paleari CD, Petucco S, Boni M, Colangeli G, Penzo M, Pessina AC (1991) Haemodynamic effects of coffee and caffeine in normal volunteers: a placebo-controlled clinical study. Journal of Internal Medicine 229: 501-504.

Chaves JF, Dworkin SF (1997) Hypnotic control of pain: historical perspectives and future prospects. International Journal of Clinical and Experimental Hypnosis 4: 356-376.

De Pascalis V, Russo O, Marrucci FS (2000) Italian norms for the Harvard Group Scale of Hypnotic Susceptibility, Form A. International Journal of Clinical and Experimental Hypnosis 48: 44-55.

Facco E, Ermani M, Rampazzo P, Tikhonoff V, Saladini M, Zanette G, Casiglia E, Spiegel D (2014) Top-down regulation of left temporal cortex by "hypnotic amusia for rhythm": a pilot study on mismatch negativity. International Journal of Clinical and Experimental Hypnosis 62: 129-144.

Facco E, Pasquali S, Zanette G, Casiglia E (2013) Hypnosis as sole anaesthesia for skin tumor removal in a patient with multiple chemical sensitivity. Anaesthesia 68: 961-965.

Facco E, Casiglia E, Masiero S, Tikhonoff V, Giacomello M, Zanette G (2011) Effects of hypnotic focused analgesia on dental pain threshold. International Journal of Clinical and Experimenal Hypnosis 59: 454-468. 
Facco E, Casiglia E, Zanette G, Masero S, Bacci C, Lapenta AM, Manani G (2009) Effects of hypnosis on dental pain threshold. Preliminary report. Pain Practice 9(Suppl.1): 47-48.

Giordano N, Tikhonoff V, Tosello MT, Casiglia E (2012) An experimental approach to hypnotic age regression. Controlled study over 10 healthy participants. Contemporary Hypnosis and Integrative Therapy 29: 271-283.

Holroyd J (1996) Hypnosis treatment of clinical pain: understanding why hypnosis is useful. International Journal of Clinical and Experimental Hypnosis 44: 33-51.

Horton J, Crawford H, Harrington G, Downs H (2004) Increased anterior corpus callosum size associated positively with hypnotisability and the ability to control pain. Brain 127: 1741-1747.

Kiernan BD, Dane JR, Phillips LH, Price DD (1995) Hypnotic analgesia reduces R-III nociceptive reflex: further evidence concerning the multifactorial nature of hypnotic analgesia. Pain 60: 39-47.

Kropotov JD, Crawford HJ, Polyakov YI (1997) Somatosensory event-related potential changes to painful stimuli during hypnotic analgesia: anterior cingulated cortex and anterior temporal cortex intracranial recordings. International Journal of Psychophysiology 27: 1-8.

Mader TJ, Blank FS, Smithline HA, Wolfe JM (2003) How reliable are pain scores? A pilot study of 20 healthy volunteers. Journal of Emergency Nursing 4: 322-325.

Melzack R, Wall PD (1965) Pain mechanisms: a new theory. Science 150: 971-979.

Priftis K, Schiff S, Tikhonoff V, Giordano N, Amodio P, Umiltà C, Casiglia E (2011) Hypnosis meets neurosciences: simulating visuospatial neglect in healthy participants. Neuropsychologia 49: 3346-3350.

Sandrini G, Milanov I, Malaguti S, Nigrelli MP, Moglia A, Nappi G (2000) Effects of hypnosis on diffuse noxius inhibitory controls. Psychology and Behavior 69: 295300.

Tikhonoff V, Azzi D, Boschetti G, Giordano N, Rempelou P, Giacomello M, Tosello MT, Facco E, Rossi AM, Lapenta AM, Regaldo G, Palatini P, Casiglia E (2012) Increase of isometric performance via hypnotic suggestion: experimental study over 10 young healthy volunteers. Contemporary Hypnosis and Integrative Therapy 29: 352-262.

Younger J (2005) The Harvard Group Scale of Hypnotic Suseptibility: accuracy of self-report and the memory for items. International Jounal of Clinical and Experimental Hypnosis 53: 306-320. 